\title{
Itinerant Ferromagnetism in a Fermi Gas of Ultracold Atoms
}

\author{
Gyu-Boong Jo ${ }^{1 *}$, Ye-Ryoung Lee ${ }^{1}$, Jae-Hoon Choi $^{1}$, Caleb A. Christensen ${ }^{1}$, \\ Tony H. Kim ${ }^{1}$, Joseph H. Thywissen ${ }^{2}$, David E. Pritchard ${ }^{1}$, and Wolfgang Ketterle ${ }^{1}$ \\ ${ }^{1}$ MIT-Harvard Center for Ultracold Atoms, Research Laboratory of Electronics, \\ Department of Physics, Massachusetts Institute of Technology, Cambridge, MA 02139, USA \\ ${ }^{2}$ Department of Physics, University of Toronto, Toronto, Ontario M5S1A7, Canada \\ *To whom correspondence should be addressed; E-mail: gyuboong@ mit.edu
}

\begin{abstract}
Can a gas of spin-up and spin-down fermions become ferromagnetic due to repulsive interactions? This question which has not yet found a definitive theoretical answer was addressed in an experiment with an ultracold twocomponent Fermi gas. The observation of non-monotonic behavior of lifetime, kinetic energy, and size for increasing repulsive interactions provides strong evidence for a phase transition to a ferromagnetic state. It implies that itinerant ferromagnetism of delocalized fermions is possible without lattice and band structure and validates the most basic model for ferromagnetism introduced by Stoner.
\end{abstract}

Magnetism is a macroscopic phenomenon with its origin deeply rooted in quantum mechanics. In condensed matter physics, there are two paradigms for magnetism: localized spins interacting via tunnelling, and delocalized spins interacting via an exchange energy. The latter gives rise to itinerant ferromagnetism which is responsible for the properties of transition metals 
like cobalt, iron and nickel. Both kinds of magnetism involve strong correlations and/or strong interactions and are not yet completely understood. For localized spins, major open questions include the interplay of magnetism with d-wave superfluidity and frustrated spin materials. For itinerant ferromagnetism (1-7), phase transition theories are still qualitative.

We implement the Stoner model, a textbook Hamiltonian for itinerant ferromagnetism (8), using a two-component gas of free fermions with short-range repulsive interactions, which can capture the essence of the screened Coulomb interaction in electron gases (8). However, there is no proof so far that this simple model for ferromagnetism is consistent when the strong interactions are treated beyond mean-field approaches. It is known that this model fails in one dimension where the ground state is singlet for arbitrary interactions, or for two particles in any dimension (3). Here, cold atoms are used to perform a quantum simulation of this model Hamiltonian in 3D and show experimentally that it leads to a ferromagnetic phase transition (2). This model is also realized in helium-3 (9), but it turns into solid and not into a ferromagnetic phase at high pressure. It has also been applied to neutrons in neutron stars (10).

So far, magnetism in ultracold gases has been studied only for spinor $(11,12)$ and dipolar (13) Bose-Einstein condensates. In these cases, magnetism is driven by weak spin-dependent interactions which nevertheless determine the structure of the condensate due to a bosonic enhancement factor. In contrast, here we simulate quantum magnetism in a strongly interacting Fermi gas.

An important recent development in cold atom science has been the realization of superfluidity and the BEC-BCS crossover in strongly interacting two-component Fermi gases near a Feshbach resonance (14). These phenomena occur for attractive interactions for negative scattering length and for bound molecules (corresponding to a positive scattering length for two unpaired atoms). Very little attention has been given to the region for atoms with strongly repulsive interactions. One reason is that this region is an excited branch, which is unstable 
against near-resonant three-body recombination into weakly-bound molecules. Nevertheless, many theoretical papers have proposed a two-component Fermi gas near a Feshbach resonance as a model system for itinerant ferromagnetism (15-22) assuming that the decay into molecules can be sufficiently suppressed. Another open question which has not been addressed is the possibility of a fundamental limit for repulsive interactions. Such a limit due to unitarity or many-body physics may be lower than the value required for the transition to a ferromagnetic state. We show that this is not the case, and that there is a window of metastability where the onset of ferromagnetism can be observed.

A simple mean-field model captures many qualitative features of the expected phase transition, but is not adequate for a quantitative description of the strongly interacting regime. The total energy of a two-component Fermi gas of average density $n$ (per spin component) in a volume $V$ is given by $E_{F} 2 V n\left[\frac{3}{10}\left\{(1+\eta)^{5 / 3}+(1-\eta)^{5 / 3}\right\}+\frac{2}{3 \pi} k_{F} a(1+\eta)(1-\eta)\right]$ where $E_{F}$ is the Fermi energy of a gas, $k_{F}$ the Fermi wavevector of a gas, $a$ the scattering length characterizing short-range interactions between the two components, and $\eta=\Delta n / n=\left(n_{1}-n_{2}\right) /\left(n_{1}+n_{2}\right)$ magnetization of the Fermi gas. The local magnetization of the Fermi gas is non-zero when the gas separates into two volumes, where the densities $n_{1}$ and $n_{2}$ of the two spin states differ by $2 \Delta n$. Note that we study an ensemble in which the number of atoms in each spin state is conserved. This is equivalent to a free electron gas at zero external magnetic field where the total magnetization is zero. The interaction term represents any short-range spin-independent potential. When the gas is fully polarized, it avoids the repulsive interaction, but increases its kinetic energy by a factor of $2^{2 / 3}$. The phase transition occurs when the minimum in energy is at nonzero magnetization (Fig. 1A) at $k_{F} a=\pi / 2$. This onset was discussed in the context of phaseseparation in a two-component Fermi gas (15-18). Fig. 1B shows several consequences of the phase transition for a system at constant pressure. First, for increasing repulsive interactions, the gas expands, lowering its density and Fermi energy; kinetic energy is therefore reduced. When 
the gas enters the ferromagnetic phase, kinetic energy increases rapidly due to the larger local density per spin state. Furthermore, the volume has a maximum value at the phase transition. This can be understood by noting that pressure in our model is $(2 / 3) E_{\text {kin }} / V+E_{\text {int }} / V$, where $E_{k i n}$ is kinetic energy and $E_{i n t}$ interaction energy. At the phase transition, the system increases its kinetic energy and reduces its interaction energy, thus reducing the pressure. This maximum in pressure at constant volume turns into a maximum in volume for a system held at constant pressure, or in a trapping potential. We have observed three predictions of this model: the onset of local magnetization through the suppression in inelastic collisions, the minimum in kinetic energy, and the maximum in the size of the cloud. These qualitative features are generic for the ferromagnetic phase transition and should be present also in more advanced models (19).

We start with an atom cloud consisting of an equal mixture of ${ }^{6} \mathrm{Li}$ atoms in the lowest two hyperfine states, held at $590 \mathrm{G}$ in an optical dipole trap with additional magnetic confinement (23). The number of atoms per spin state $\sim 6.5 \times 10^{5}$ corresponds to a Fermi temperature $T_{F}$ of $\sim 1.4 \mu \mathrm{K}$. The effective temperature $T$ could be varied between $T / T_{F}=0.1$ and $T / T_{F}=0.6$ and was determined right after the field ramp by fitting the spatial distribution of the cloud with a finite temperature Thomas-Fermi profile. Note that $k_{F}^{\circ}$ is the Fermi wavevector of the non-interacting gas calculated at the trap center. Applying the procedure discussed in Ref. (24) to repulsive interactions, we estimate that the real temperature is $\sim 20 \%$ larger than the effective one. The effective temperature did not depend on $k_{F}^{\circ} a$ for $k_{F}^{\circ} a<6$. At higher temperatures, additional shot-to-shot noise was caused by large fluctuations in the atom number. From the starting point at $590 \mathrm{G}$, the magnetic field was increased towards the Feshbach resonance at $834 \mathrm{G}$, thus providing adjustable repulsive interactions. Due to the limited lifetime of the strongly interacting gas, it was necessary to apply the fastest possible field ramp, limited to $4.5 \mathrm{~ms}$ by eddy currents. The ramp time is approximately equal to the inverse of the axial trap frequency (23) and therefore only marginally adiabatic. Depending on the magnetic field 
during observation, either atoms or atoms and molecules were detected by absorption imaging as described in Fig. S1 (25).

The emergence of local spin polarization can be observed by the suppression of (either elastic or inelastic) collisions, as the Pauli exclusion principle forbids collisions in a fully polarized cloud. We monitor inelastic three-body collisions which convert atoms into molecules. The rate (per atom) is proportional to $f(a, T) n_{1} n_{2}$ or $f(a, T) n^{2}\left(1-\eta^{2}\right)$ and is therefore a measure of the magnetization $\eta$. For $k_{F} a \ll 1$, the rate coefficient $f(a, T)$ is proportional to $a^{6} \max \left(T, T_{F}\right)(26)$. This rate can be observed by monitoring the initial drop in the number of atoms during the first $2 \mathrm{~ms}$ after the field ramp. We avoided longer observation times since the increasing molecule fraction could modify the properties of the sample.

Fig. 2 shows a sharp peak in the atom loss rate around $k_{F}^{\circ} a \simeq 2.5$ at $T / T_{F}=0.12$ indicating a transition in the sample to a state with local magnetization. The gradual decrease is consistent with the inhomogeneous density of the cloud where the transition occurs first in the center. The large suppression of the loss rate indicates a large local magnetization of the cloud.

The kinetic energy of the cloud was determined by suddenly switching off the optical trap and the Feshbach fields right after the field ramp and then imaging state $|1\rangle$ atoms at zero field using the cycling transition after a ballistic expansion time of $\triangle_{\text {tof }}=4.6 \mathrm{~ms}$. The kinetic energy was obtained from the Gaussian radial width $\sigma_{x}$ as $E_{k i n}=\frac{3 m \sigma_{x}^{2}}{2 \triangle_{t o f}{ }^{2}}$ where $m$ is the mass of the ${ }^{6} \mathrm{Li}$ atom. Fig. 3 demonstrates a minimum of the kinetic energy at $k_{F}^{\circ} a \simeq 2.2$ for the coldest temperature, $T / T_{F}=0.12$, nearly coinciding with the onset of local polarization. The peak in the atom loss rate occurs slightly later than the minimum of kinetic energy, probably because $f(a, T)$ increases with $a(22)$. Since the temperature did not change around $k_{F}^{\circ} a \simeq 2.2$, the increase in kinetic energy is not caused by heating, but by a sudden change in the properties of the gas, consistent with the onset of ferromagnetism. The observed increase in kinetic energy is $\sim 20 \%$ at $T / T_{F}=0.12$, smaller than the value $\left(2^{2 / 3}-1\right)=0.59$ predicted for a fully polarized 
gas. This discrepancy could be due to the absence of polarization or partial polarization in the wings of the cloud. Also, it is possible that the measured kinetic energy of the strongly interacting gas before the phase transition includes some interaction energy if the Feshbach fields are not suddenly switched off. For the current switch-off time of $\sim 100 \mu \mathrm{s}$, this should be only a $5 \%$ effect, but the magnetic field decay may be slower due to eddy currents.

Finally Fig. 4 shows our observation of a maximum cloud size at the phase transition, in agreement with the prediction of the model. The cloud size may not have fully equilibrated since our ramp time was only marginally adiabatic, but this alone cannot explain the observed maximum.

The suppression of the atom loss rate, the minimum in kinetic energy, and the maximum in cloud size show a strong temperature dependence between $T / T_{F}$ of 0.12 and 0.22 . As the properties of a normal Fermi gas approaching the unitarity limit with $k_{F}^{\circ} a>>1$ should be insensitive to temperature variations in this range, this provides further evidence for a transition to a new phase.

At higher temperature (e.g. $T / T_{F}=0.39$ in Fig. 3), the observed non-monotonic behavior becomes less pronounced and shifts to larger values of $k_{F}^{\circ} a$ for $3 \leq k_{F}^{\circ} a \leq 6$. For all three observed properties (Figs. 2-4), a nonmonotonic behavior is no longer observed at $T / T_{F}=$ 0.55 (27). One interpretation is that at this temperature and above, there is no phase transition any more. Note that in a mean-field approximation, a ferromagnetic phase would appear at all temperatures, but for increasing values of $k_{F}^{\circ} a$. Our observations may imply that the interaction energy saturates around $k_{F}^{\circ} a \approx 5$.

The spin-polarized ferromagnetic state should not suffer from inelastic collisions. However, typical lifetime were 10 - $20 \mathrm{~ms}$, probably related to a small domain size (see below) and threebody recombination at domain walls.

We were unsuccessful in imaging ferromagnetic domains using differential in-situ phase- 
contrast imaging (28). A noise level of $S / N \sim 10$ suggests that there were at least 100 domains in a volume given by our spatial resolution of $\sim 3 \mu \mathrm{m}$ and the radial size of the cloud. This implies that the maximum volume of the spin domains is $\sim 5 \mu \mathrm{m}^{3}$, containing $\sim 50$ spinpolarized atoms. We suspect that the short lifetime prevented the domains from growing to a larger size, and eventually adopting the equilibrium texture of the ground state, which has been predicted to have the spins pointing radially outward, like a hedgehog $(20,22)$. All our measurements are sensitive only to local spin polarization, independent of domain structure and texture.

The only difference between our experiment and the ideal Stoner model is a molecular admixture of $25 \%$ (Fig. 4). The molecular fraction was constant for $k_{F}^{\circ} a>1.8$ for all temperatures and therefore cannot be responsible for the sudden change of behavior of the gas at $k_{F}^{\circ} a \simeq 2.2$ for the coldest temperature $T / T_{F}=0.12$. This was confirmed by repeating the kinetic energy measurements with a molecular admixture of $60 \%$. The minimum in the kinetic energy occurred at the same $k_{F}^{\circ} a$ within experimental accuracy.

Before we can compare the observed phase transition at $k_{F}^{\circ} a \simeq 2.2$ to the theoretical predictions, we have to replace the ideal gas $k_{F}^{\circ}$ by the value for the interacting gas, which is smaller by $\sim 15 \%$ because of the expansion of the cloud (Fig. 4 ), and obtain a critical value for $k_{F} a \simeq 1.9 \pm 0.2$. At $T / T_{F}=0.12$, the finite temperature correction in the critical value for $k_{F} a$ is predicted to be less than $5 \%(19)$. The observed value for $k_{F} a$ is larger than the mean-field prediction of $\pi / 2$ and the second order prediction of 1.054 at zero temperature (19). Depending on the theoretical approach, the phase transition has been predicted to be first or second order. This could not been discerned in our experiment due to the inhomogeneous density of the cloud.

Ref. (19) speculated that earlier experiments on the measurement of the interaction energy (29) and RF spectroscopy of Fermi gases (30) showed evidence for the transition to a 
ferromagnetic state at or below $k_{F} a=1$. This interpretation is ruled out by our experiment.

Our work demonstrates a remarkable asymmetry between positive and negative scattering length. Early work (15) predicted that for $k_{F}|a|=\pi / 2$, both an attractive and a repulsive Fermi gas become mechanically unstable (against collapse, and phase separation, respectively). In an attractive Fermi gas, however, the mechanical instability does not occur (due to pairing (31)), in contrast to our observations in a repulsive Fermi gas. This suggests that the maximum total repulsive energy (in units of $3 / 5(2 V n) E_{F}$ ) is larger than the maximum attractive energy $|\beta|$ of 0.59 (32) realized for infinite $a(23)$.

Heisenberg's explanation for ferromagnetism was based on exchange energy, i.e. the Pauli principle and spin-independent repulsive interactions between the electrons. However, it remained an open question, what other "ingredients" were needed for itinerant ferromagnetism. It was only in $1995(6,7)$, that a rigorous proof was given that, in certain lattices, spin-independent Coulomb interactions can give rise to ferromagnetism in itinerant electron systems. Our finding implies that Heisenberg's idea does not require a lattice and band structure, but applies already to a free gas with short-range interactions. Our experiment can be regarded as quantum simulation of a Hamiltonian for which even the existence of a phase transition was unproven. This underlines the potential of cold atom experiments as quantum simulators for many-body physics. 
A

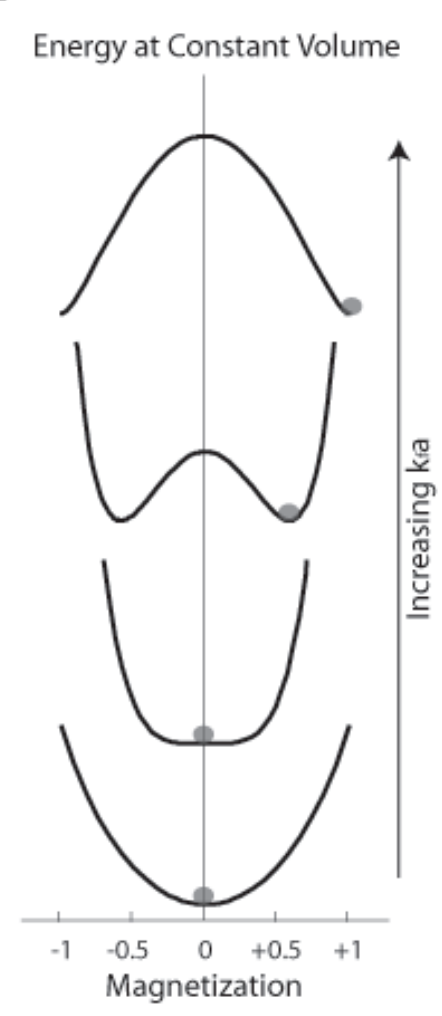

B

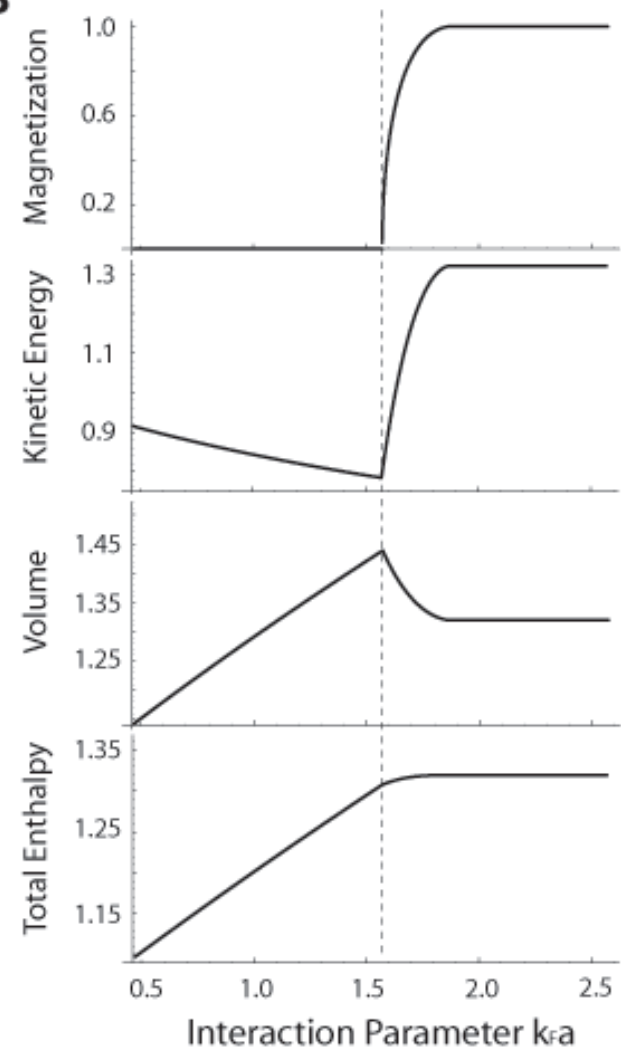

Figure 1: Ferromagnetic phase transition at $\mathrm{T}=0$, according to the mean-field model described in the text. The onset of itinerant ferromagnetism occurs when the energy as a function of magnetization flips from a U-shape to a W-shape (A). Figure (B) shows the enthalpy, volume and kinetic energy (normalized to their values for the ideal Fermi gas), and magnetization as a function of the interaction parameter $k_{F} a$. Note that $k_{F}$ is defined by the density of the gas. The dotted line marks the phase transition. 


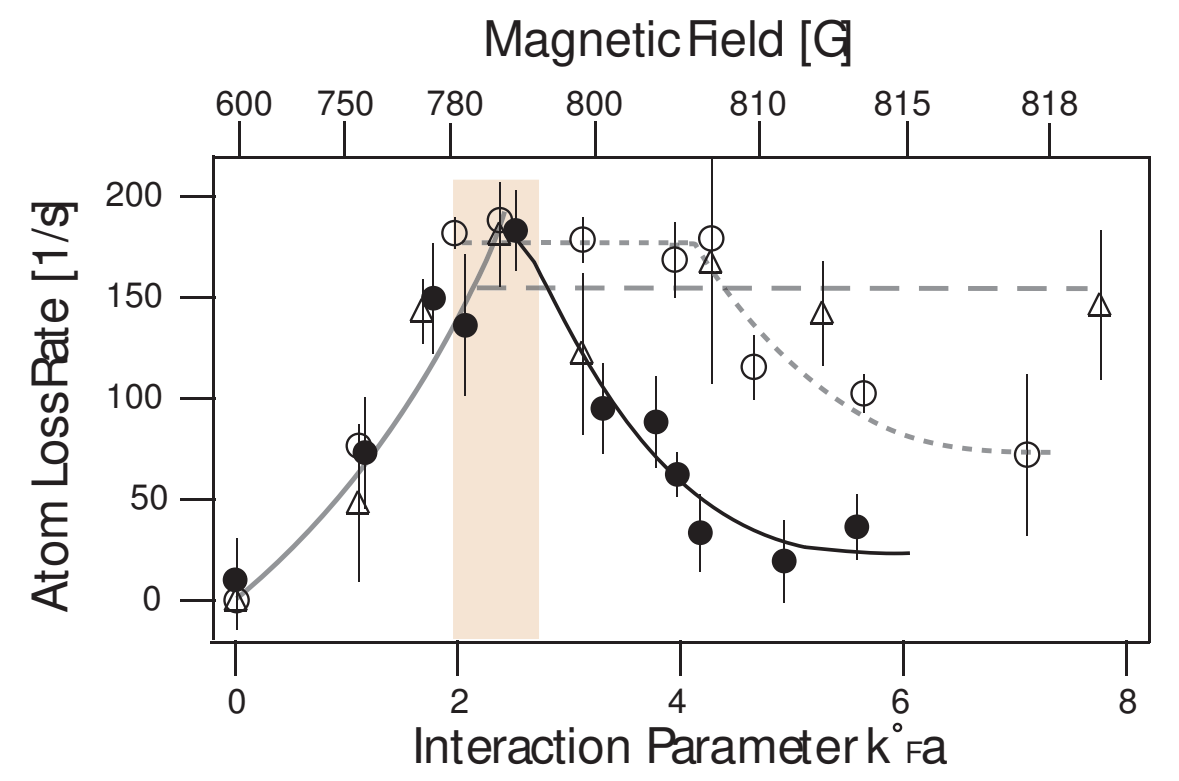

Figure 2: Atom loss rate as a probe for local spin polarization, for different temperatures. (a) $T / T_{F}=0.55$ (dashed curve), (b) $T / T_{F}=0.22$ (dotted curve), and $T / T_{F}=0.12$ (solid black curve). The curves are guides to the eye, based on the assumption of a loss rate which saturates for increasing $a$ in the normal state. The shaded area around the phase transition at $T / T_{F}=0.12$ highlights the same region as in Figs. 3 and 4. 


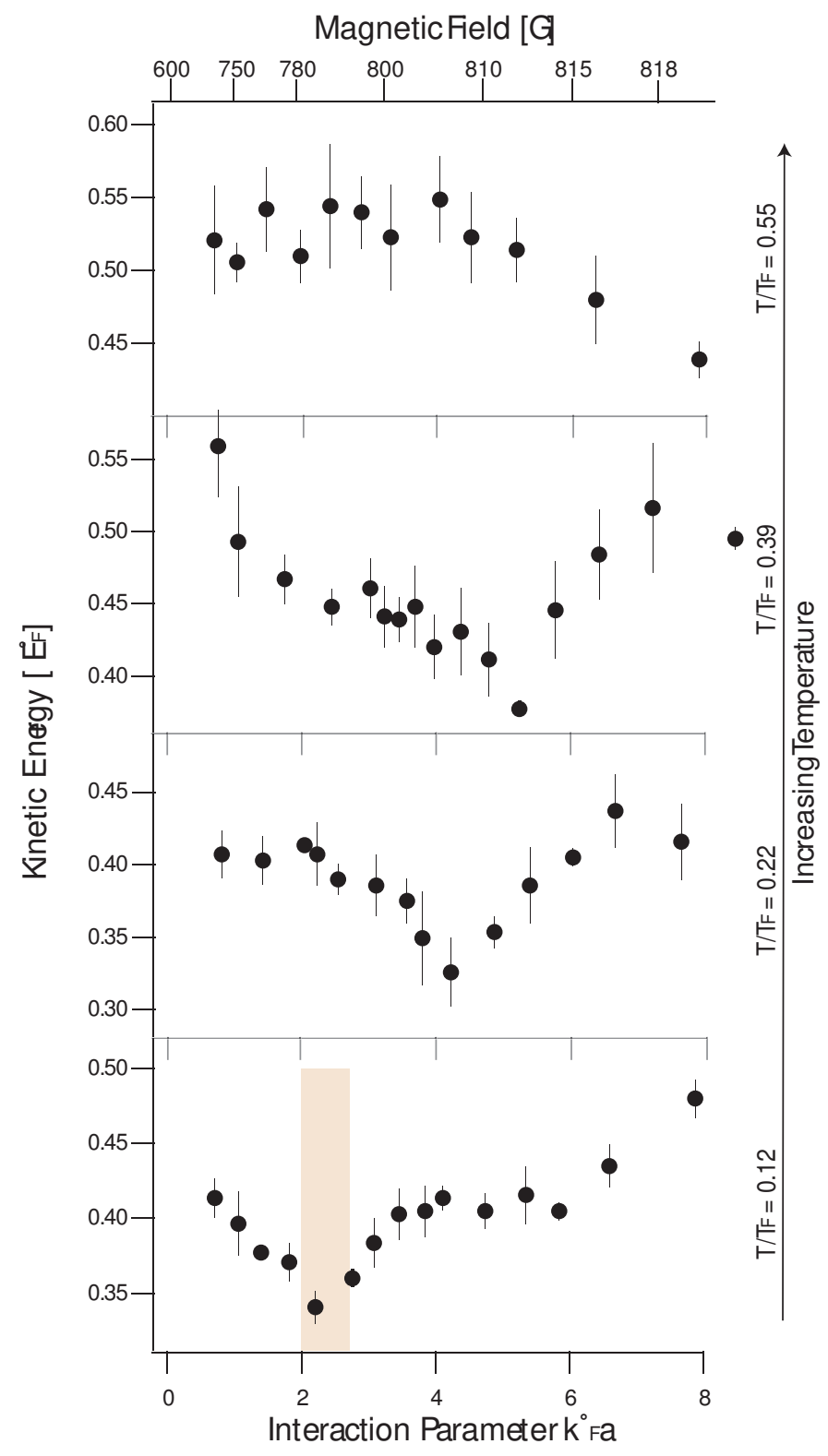

Figure 3: Kinetic energy of a repulsively interacting Fermi gas determined for different interaction parameters $k_{F}^{\circ} a$ and temperatures. The measured kinetic energy is normalized by the Fermi energy $E_{F}^{\circ}$ of the noninteracting Fermi gas at $T=0$, calculated at the trap center with the same number of atoms per spin state. Each data point represents the average of three or four measurements. 


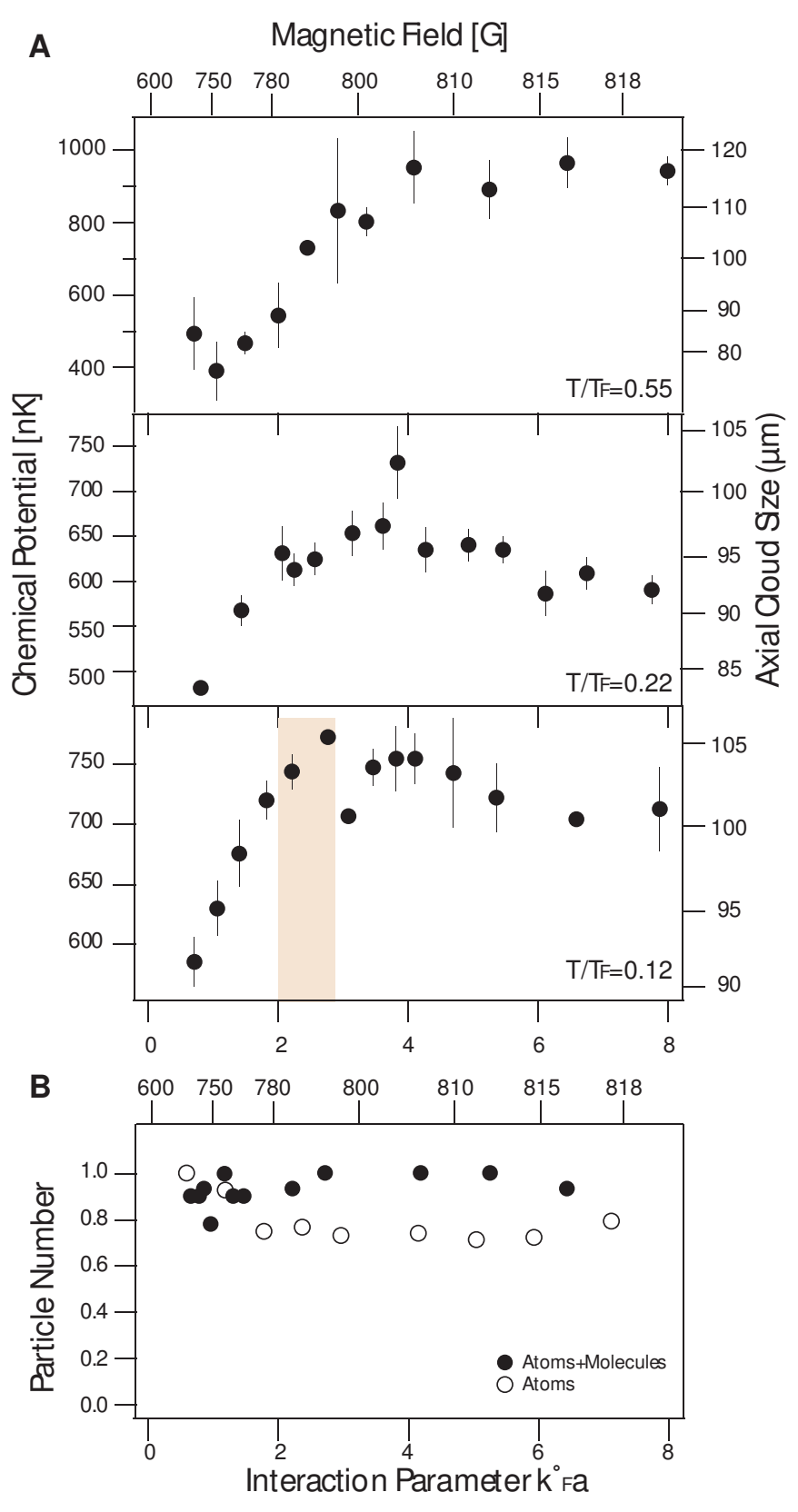

Figure 4: Maximum in volume at the phase transition (A) Axial size and chemical potential of the cloud for various temperatures. The chemical potential $\mu$ is determined from the measured cloud size, $\sigma_{z}$ as $\mu=\frac{1}{2} m \omega_{z}{ }^{2} \sigma_{z}{ }^{2}$ (B) Number of particles including both atoms and molecules right after the field ramp. This result shows that $25 \%$ of atoms were converted into molecules during the field ramp, and this fraction stayed constant for $k_{F}^{\circ} a>1.8$, where the phase transition was reached. This molecule fraction was independent of temperature. 


\section{References and Notes}

1. F. Bloch, Z.Phys. 57, 545 (1929).

2. E. Stoner, Phil. Mag. 15, 1018 (1933).

3. E. Lieb, D. Mattis, Phys. Rev. 125, 164 (1962).

4. P. W. Brouwer, Y. Oreg, B. I. Halperin, Phys. Rev. B 60, R13977 (1999).

5. D. Vollhardt, N. Blumer, K. Held, M. Kollar, Metallic Ferromagnetism - An Electronic Correlation Phenomenon. Lecture Notes in Physics, Vol. 580, Springer, Heidelberg, 2001.

6. H. Tasaki, Phys. Rev. Lett. 75, 4678 (1995).

7. A. Tanaka, H. Tasaki, Phys. Rev. Lett. 98, 116402 (2007).

8. D. W. Snoke, Solid state physics:Essential concepts (Addison-Wesley, San Francisco, 2008).

9. D. Vollhardt, Rev. Mod. Phys. 56, 99 (1984).

10. J. Pfarr, Z.Phys. 251, 152 (1972).

11. J. Stenger, et al., Nature 396, 345 (1998).

12. L. E. Sadler, J. M. Higbie, S. R. Leslie, M. Vengalattore, D. M. Stamper-Kurn, Nature 443, 312 (2006).

13. T. Lahaye, et al., Nature 448, 672 (2007).

14. M. Inguscio, W. Ketterle, C. Salomon, Ultracold Fermi Gases, Proceedings of the International School of Physics "Enrico Fermi”, Course CLXIV (IOS Press, Amsterdam, 2008). 
15. M. Houbiers, et al., Phys. Rev. A 56, 4864 (1997).

16. L. Salasnich, B. Pozzi, A. Parola, L. Reatto, J. Phys. B: At. Mol. Opt. Phys. 33, 3943 (2000).

17. M. Amoruso, I. Meccoli, A. Minguzzi, M. Tosi, Eur. Phys. J. D 8, 361 (2000).

18. T. Sogo, H. Yabu, Phys. Rev. A 66, 043611 (2002).

19. R. A. Duine, A. H. MacDonald, Phys. Rev. Lett. 95, 230403 (2005).

20. I. Berdnikov, P. Coleman, S. H. Simon, Phys. Rev. B 79, 224403 (2009).

21. S. Zhang, H. Hung, C. Wu, arXiv:0805.3031v4 (2008).

22. L. J. LeBlanc, J. H. Thywissen, A. A. Burkov, A. Paramekanti, Phys. Rev. A 80, 013607 (2009).

23. See supporting material on Science Online.

24. J. Kinast, et al., Science 307, 1296 (2005).

25. M. W. Zwierlein, et al., Phys. Rev. Lett. 91, 250401 (2003).

26. J. P. D’Incao, B. D. Esry, Phys. Rev. Lett. 94, 213201 (2005).

27. The interpretation of the loss rate is complicated since $f(a, T)$ is unkown for $k_{F} a \geq 1$. The three body rate $f(a, T)$ is expected to be unitarity saturated for $k_{F} a \gg 1$ (33). The lines in Fig. 2 indicate that the observed loss rate is consistent with unitarity saturation and a sudden drop at the phase transition, which occurs at large values of $k_{F} a$ at higher temperature.

28. Y. Shin, M. W. Zwierlein, C. H. Schunck, A. Schirotzek, W. Ketterle, Phy. Rev. Lett. 97, $030401(2006)$.

29. T. Bourdel, et al., Phys. Rev. Lett. 91, 020402 (2003). 
30. S. Gupta, et al., Science 300, 1723 (2003).

31. P. Nozières, S. Schmitt-Rink, J. Low Temp. Phys. 59, 1 (1985).

32. J. Carlson, S. Reddy, Phys. Rev. Lett. 95, 060401 (2005).

33. T. Weber, J. Herbig, M. Mark, H.-C. Nägerl, R. Grimm, Phys. Rev. Lett. 91, 123201 (2003).

34. This work was supported by the NSF and ONR, through a MURI program, and under ARO Grant No. W911NF-07-1-0493 with funds from the DARPA OLE program. G.-B. Jo and Y.-R. Lee acknowledge additional support from the Samsung Foundation. We would like to thank E. Demler, W. Hofstetter, A. Paramekanti, L. J. LeBlanc, and G.J. Conduit for useful discussions, T. Wang for experimental assistance, and A. Keshet for development of the computer control system. 


\section{Supporting materials: Itinerant Ferromagnetism in a Fermi Gas of Ultracold Atoms}

\section{Materials and Methods}

Preparation of the ultracold ${ }^{6} \mathbf{L i}$ cloud $\quad$ The first step is the production of a spin-polarized Fermi gas in the $\left|F=3 / 2, m_{F}=3 / 2\right\rangle$ state by sympathetic cooling with bosonic ${ }^{23} \mathrm{Na}$ atoms in a magnetic trap as described in ref $(S 1)$. The ${ }^{6} \mathrm{Li}$ cloud was then loaded into a deep optical dipole trap with a maxium power of $3 \mathrm{~W}$ and radial trap frequency of $\sim 3.0 \mathrm{kHz}$, followed by an $\mathrm{RF}$ transfer into the lowest hyperfine state $\left|F=1 / 2, m_{F}=1 / 2\right\rangle$. Additional axial confinement was provided by magnetic fields. An equal mixture of $|1\rangle$ and $|2\rangle$ spin states (corresponding to the $\left|F=1 / 2, m_{F}=1 / 2\right\rangle$ and $\left|F=1 / 2, m_{F}=-1 / 2\right\rangle$ states at low magnetic field) was prepared by a Landau-Zener RF sweep at a magnetic field of $590 \mathrm{G}$, followed by $1 \mathrm{~s}$ for decoherence and further evaporative cooling at $300 \mathrm{G}$. Finally, the optical trapping potential was adiabatically reduced over $600 \mathrm{~ms}$, and the field increased back to $590 \mathrm{G}$. The trap had a depth of $7.1 \mu \mathrm{K}$ and was nearly cigar shaped with frequencies $\nu_{x}=\nu_{y} \simeq 300 \mathrm{~Hz}$ and $\nu_{z} \simeq 70 \mathrm{~Hz}$.

\section{Supporting online text}

Estimation of the maximum total repulsive energy Full phase separation at zero temperature requires a total repulsive energy of $\left(2^{2 / 3}-1\right)=0.59$ in units of $3 / 5(2 V n) E_{F}$. At finite temperature $T$, one has to add $T S$ where $S=(2 V n) k_{B} \ln 2$ is the entropy difference between the two phases. Our tentative observation of a ferromagnetic phase at $T=0.39 T_{F}$ implies a repulsive energy of $\sim 1.04$ assuming full phase separation, larger than the maximum attraction energy of 0.59 . 


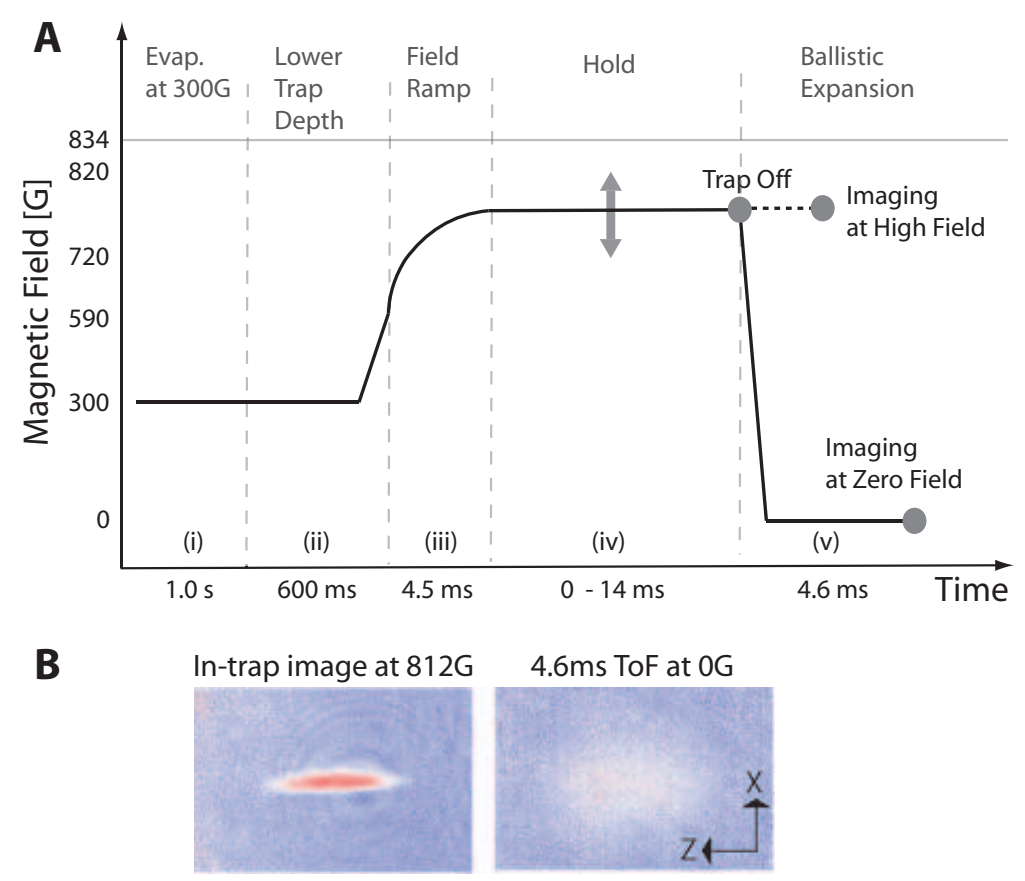

Fig. S1: (A) The schematic shows the time sequence of the experiment. The sample was exposed to the magnetic field of interest for 0 - $14 \mathrm{~ms}$ and analyzed in-situ for loss measurement or after $4.6 \mathrm{~ms}$ time-of-flight for the measurement of kinetic energy and the axial size of the cloud. The Feshbach fields were suddenly switched off at a rate of $1 \mathrm{G} / \mu \mathrm{s}$, preventing the conversion of interaction energy into kinetic energy during the expansion. (B) This absorption image shows the $|1\rangle$ component of the cloud trapped at $812 \mathrm{G}$ (left), and after $4.6 \mathrm{~ms}$ ballistic expansion imaged at zero field (right). The field of view is $840 \mu \mathrm{m} \times 550 \mu \mathrm{m}$. The magnetic field ramp was limited by eddy currents to $4.5 \mathrm{~ms}$. Spectroscopic measurements of the magnetic field showed that the field was trailing behind the current which was controlled with a time constant faster than $1 \mathrm{~ms}$.

\section{References and Notes}

S1. Z. Hadzibabic, et al., Phys. Rev. Lett. 91, 160401 (2003). 